\title{
Effects of horizontal acceleration on the superconducting gravimeter CT \#036 at Ishigakijima, Japan
}

\author{
Yuichi Imanishi ${ }^{*}$ (D), Kazunari Nawa ${ }^{2}$, Yoshiaki Tamura ${ }^{3}$ and Hiroshi Ikeda ${ }^{4}$
}

\begin{abstract}
In the gravity sensor of a superconducting gravimeter, a superconducting sphere as a test mass is levitated in a magnetic field. Such a sensor is susceptible to applied horizontal as well as vertical acceleration, because the translational degrees of freedom of the mass are not perfectly limited to the vertical direction. In the case of the superconducting gravimeter CT \#036 installed at Ishigakijima, Japan, horizontal ground acceleration excited by the movements of a nearby VLBI antenna induces systematic step noise within the gravity recordings. We investigate this effect in terms of the static and dynamic properties of the gravity sensor using data from a collocated seismometer. It is shown that this effect can be effectively modeled by the coupling between the horizontal and vertical components in the gravity sensor. It is also found that the mechanical eigenfrequency for horizontal translation of the levitating sphere is approximately $3 \mathrm{~Hz}$.
\end{abstract}

Keywords: Superconducting gravimeter, Ishigakijima, VLBI, Magnetic suspension, Horizontal acceleration

\section{Introduction}

In the superconducting gravimeter (SG) (Prothero and Goodkind 1968; Goodkind 1999; Hinderer et al. 2015), the elastic restoring force as in mechanical spring-type gravimeters is replaced with a magnetic levitation force exerted on a superconductor. As shown in Fig. 1, there are two main superconducting coils in the gravity sensor, and the sphere is located near the center of the upper coil. Most of the levitating force is provided by the lower coil, whereas most of the horizontal restoring force is provided by the upper coil. Conceptually, the mass is confined by a weak spring in the vertical direction and by a strong spring in the horizontal direction. If these degrees of freedom are ideally independent, the observed gravity signal is insensitive to horizontal acceleration applied to the instrument. In reality, however, the magnetic levitation force decreases as the sphere is displaced away from the central axis of the supporting

\footnotetext{
*Correspondence: imanishi@eri.u-tokyo.ac.jp

1 Earthquake Research Institute, The University of Tokyo, 1-1-1, Yayoi,

Bunkyo, Tokyo 113-0032, Japan

Full list of author information is available at the end of the article
}

magnetic field generated by the coils, implying that coupling exists between horizontal and vertical components. This effect is utilized when we adjust the orientation of the gravimeter to the local vertical. Under normal operation of the SG, its orientation is automatically controlled by a tilt compensation device called thermal levelers, so that the coupling does not affect long-period gravity signals. However, large horizontal acceleration applied to the instrument would cause finite movements of the test mass in the horizontal directions and therefore affect the gravity signal through the coupling with the vertical component.

In February 2012, we installed an SG (CT \#036) in the VERA (VLBI exploration of radio astrometry) Ishigakijima station, Japan (Fig. 2), belonging to the National Astronomical Observatory of Japan, with the main purpose of detecting possible gravity signals associated with long-term slow slip events occurring beneath the Yaeyama Islands (Heki and Kataoka 2008). This gravimeter had been in operation until 2011 at the Inuyama Observatory of Nagoya University (e.g., Nawa et al. 2009). Ikeda et al. (2013) described the preparatory work for the gravimeter before its movement to the Ishigakijima 


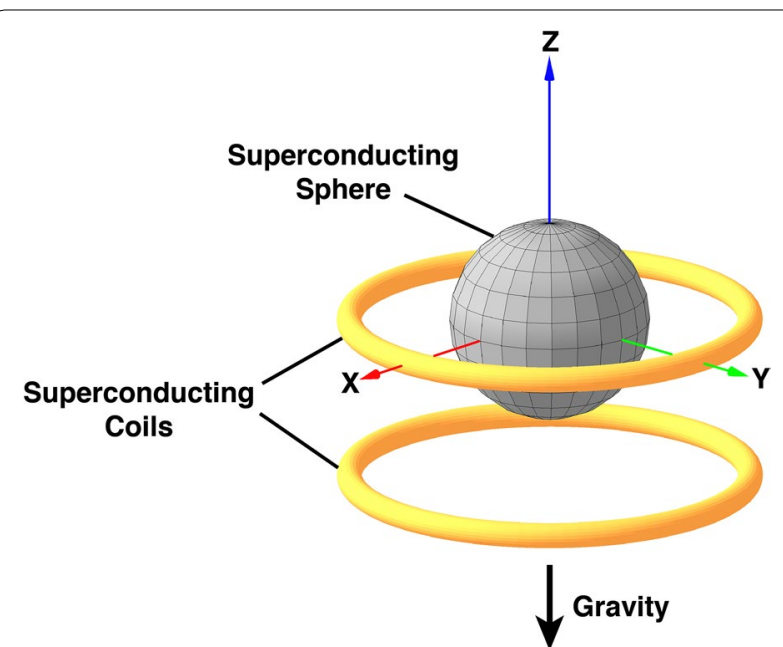

Fig. 1 Superconducting sphere and coils in the gravity sensor of the superconducting gravimeter

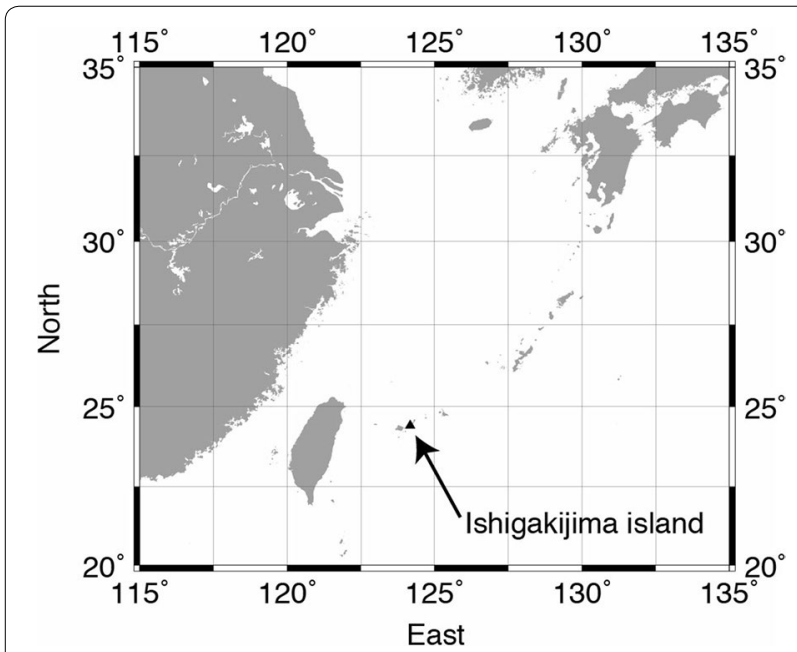

Fig. 2 Location of Ishigakijima Island, Japan, where the superconducting gravimeter $\mathrm{CT} \# 036$ was installed

Island. As soon as we started gravity observations with the SG at Ishigakijima, we learned that the station had several types of observational problems that we were not very familiar with. One such problem was observational noise from the VLBI antenna. The VERA Ishigakijima station, which is equipped with a 20-m-diameter parabolic antenna, is one of four VLBI stations that belong to the VERA project (e.g., Honma et al. 2000). The distance between the VLBI antenna and the SG is approximately $35 \mathrm{~m}$. When the antenna begins to move, step noise (gravity increasing) is recorded in the gravity signal (Fig. 3). The gravity recovers its previous value when the antenna stops. At first, we suspected that some electrical interference from the antenna system was the cause of the noise, but operating the gravimeter system with batteries (i.e., electrically isolating the gravimeter from the antenna) did not eliminate the noise, and so it was verified that horizontal ground vibrations caused by the movements of the antenna were responsible. The existence of such noise does not pose a fatal problem for the purpose of studying long-term gravity changes at this station, because we have information on exactly when the noise is generated. However, step noise in general can have power in a wide frequency range and may affect the noise level of the SG in the tidal to seismic bands (Rosat et al. 2004). Therefore, in this paper, we investigate this effect in a systematic way from the viewpoint of a gravity sensor based on magnetic suspension.

\section{Effects of movement of the VLBI antenna on the gravimeter}

Static effects of horizontal acceleration

Let us consider that a superconducting sphere with a mass $m$ is levitated inside the gravity sensor of the SG. The sphere is balanced at a position where the upward force from the supporting magnetic field is equal in magnitude to the downward gravity force. We take a cylindrical coordinate system $(r, \theta, z)$ whose origin $O$ coincides with the average position of the sphere. Let $z$ be upward positive, and let $U$ be the potential sensed by the sphere. $U$ takes a minimum at the balancing point. Assuming that the supporting magnetic field is cylindrically symmetric with respect to the vertical axis, $U$ is expanded in a Taylor series around $O$ as follows:

$$
\begin{aligned}
U(r, z)= & U(0,0)+\left.r \frac{\partial U}{\partial r}\right|_{O}+\left.z \frac{\partial U}{\partial z}\right|_{O} \\
& +\frac{1}{2}\left[\left.r^{2} \frac{\partial^{2} U}{\partial r^{2}}\right|_{O}+\left.2 r z \frac{\partial^{2} U}{\partial r \partial z}\right|_{O}+\left.z^{2} \frac{\partial^{2} U}{\partial z^{2}}\right|_{O}\right] \\
& +\frac{1}{6}\left[\left.r^{3} \frac{\partial^{3} U}{\partial r^{3}}\right|_{O}+\left.3 r^{2} z \frac{\partial^{3} U}{\partial r^{2} \partial z}\right|_{O}\right. \\
& \left.+\left.3 r z^{2} \frac{\partial^{3} U}{\partial r \partial z^{2}}\right|_{O}+\left.z^{3} \frac{\partial^{3} U}{\partial z^{3}}\right|_{O}\right]+\cdots
\end{aligned}
$$

where the subscript $O$ denotes the evaluation of the differential at the origin of the coordinate system. In a real instrument, a feedback control is in effect to hold the sphere in a fixed vertical position. Here, we consider only the mechanical properties, and the effect of feedback is discussed separately.

The superconducting sphere levitated inside the gravity sensor of the SG has rotational as well as translational degrees of freedom. By analyzing the instrumental noise inherent to the SG, Imanishi $(2005,2009)$ showed that the well-known long-period parasitic mode is likely the rotational motion of the sphere around its center 


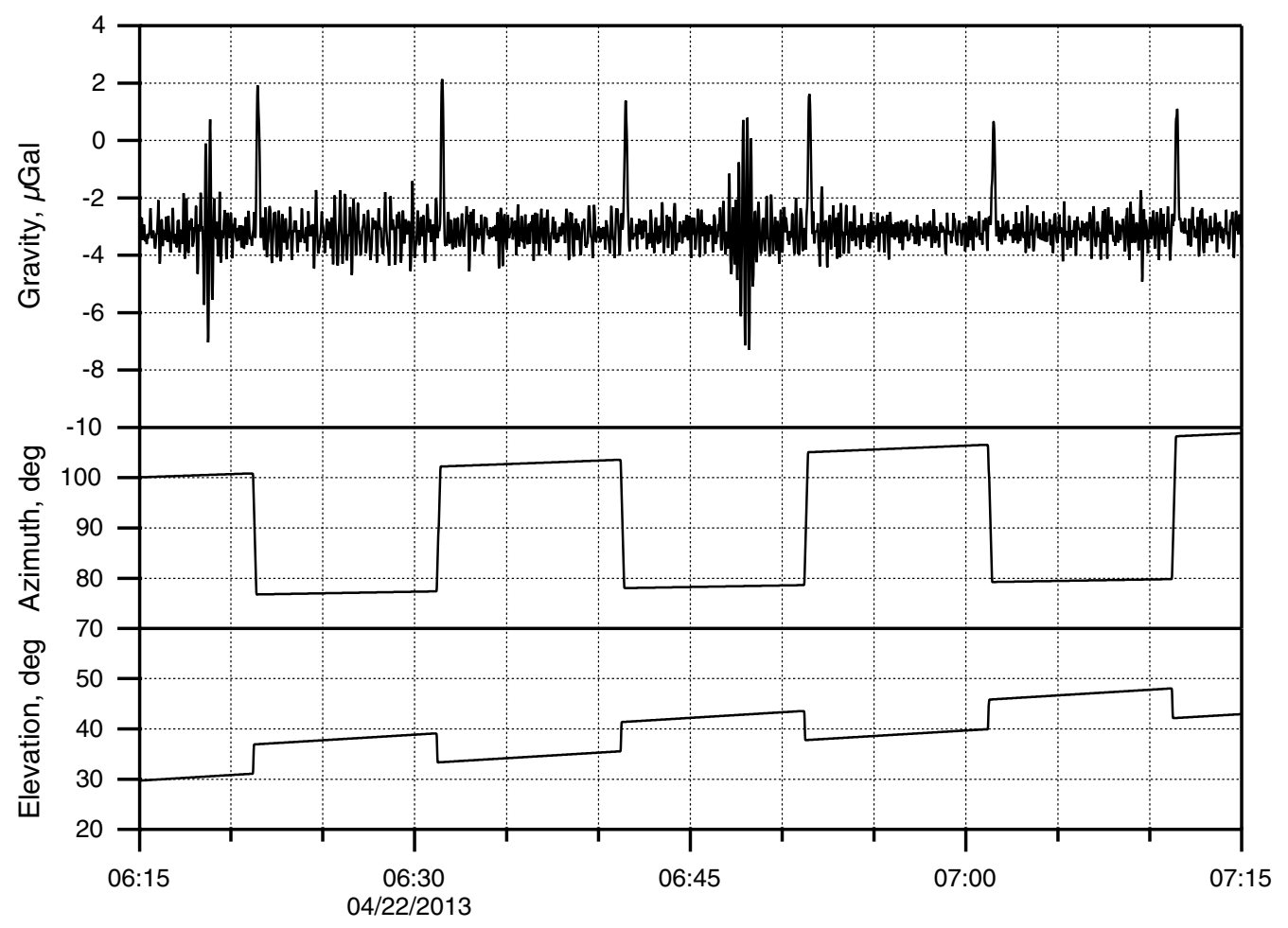

Time, UTC

Fig. 3 Typical effects of the movement of the VLBI antenna on the superconducting gravimeter at the VERA Ishigakijima station

of gravity. Although the existence of this mode implies a weak dependence of the potential on the rotational angles of the sphere, here we consider only translational degrees of freedom and neglect rotational ones.

In Eq. (1),

$$
\begin{aligned}
& \left.\frac{\partial U}{\partial r}\right|_{O}=0 \\
& \left.\frac{\partial U}{\partial z}\right|_{O}=0
\end{aligned}
$$

must be satisfied so that the sphere is balanced at $O$ Additionally, by imposing a condition that $U$ is infinitely differentiable at $O$, the terms in the odd-order powers of $r$ must vanish. Therefore, retaining the terms up to the third-order differentiation with respect to $r$ and $z$, we have

$$
\begin{aligned}
U(r, z)= & U(0,0)+\frac{1}{2}\left(\alpha_{\mathrm{H}} r^{2}+\alpha_{\mathrm{V}} z^{2}\right) \\
& +\frac{1}{6}\left(3 \beta_{\mathrm{H}} r^{2} z+\beta_{\mathrm{V}} z^{3}\right)
\end{aligned}
$$

where

$$
\alpha_{\mathrm{H}}=\left.\frac{\partial^{2} U}{\partial r^{2}}\right|_{O}
$$

$$
\begin{gathered}
\alpha_{\mathrm{V}}=\left.\frac{\partial^{2} U}{\partial z^{2}}\right|_{O} \\
\beta_{\mathrm{H}}=\left.\frac{\partial^{3} U}{\partial r^{2} \partial z}\right|_{O} \\
\beta_{\mathrm{V}}=\left.\frac{\partial^{3} U}{\partial z^{3}}\right|_{O}
\end{gathered}
$$

The coefficients of the second-order terms of the potential $\alpha_{\mathrm{H}}$ and $\alpha_{\mathrm{V}}$ are the "spring constants" in the horizontal and vertical directions, respectively. $\alpha_{\mathrm{H}}>0$ and $\alpha_{\mathrm{V}}>0$ must hold so that $U$ takes a minimum at the balancing position. The sphere is supported by a weak spring in the vertical direction and by a strong spring in the horizontal direction; otherwise, the sphere would very easily move away from the central axis of the coils. Therefore, $\alpha_{\mathrm{H}} \gg \alpha_{\mathrm{V}}$. The (angular) eigenfrequencies for vibrations in small amplitude (as simple harmonic oscillators) are given by

$$
\omega_{\mathrm{H}}=\sqrt{\frac{\alpha_{\mathrm{H}}}{m}}
$$




$$
\omega_{\mathrm{V}}=\sqrt{\frac{\alpha_{\mathrm{V}}}{m}}
$$

in the horizontal and vertical directions, respectively. It follows from $\alpha_{\mathrm{H}} \gg \alpha_{\mathrm{V}}$ that $\omega_{\mathrm{H}} \gg \omega_{\mathrm{V}}$.

The coefficients of the third-order terms of the potential $\beta_{\mathrm{H}}$ and $\beta_{\mathrm{V}}$ denote the deviation from a purely harmonic potential. $\beta_{\mathrm{H}}$ is the coefficient of the term denoting the coupling between the horizontal and vertical components. $\beta_{\mathrm{V}}$ is the coefficient of the higher-order term for the vertical component. In an ideal gravity sensor, both $\beta_{\mathrm{H}}=0$ and $\beta_{\mathrm{V}}=0$ would be satisfied; in reality, however, these coefficients are finite.

The restoring forces on the sphere for this potential are given by

$$
\begin{aligned}
& F_{\mathrm{r}}=-\frac{\partial U}{\partial r}=-\alpha_{\mathrm{H}} r-\beta_{\mathrm{H}} r z \\
& F_{z}=-\frac{\partial U}{\partial z}=-\alpha_{\mathrm{V}} z-\frac{1}{2} \beta_{\mathrm{H}} r^{2}-\frac{1}{2} \beta_{\mathrm{V}} z^{2}
\end{aligned}
$$

in the radial and vertical directions, respectively.

When we adjust the vertical orientation of the gravimeter, we seek an extremum of the gravity output by tilting the instrument so that the direction of the gravity sensor exactly coincides with the local vertical. Experimentally, the change in the gravity output $\Delta g$ depends quadratically on the tilt angle, and thus,

$$
\Delta g=c \varphi^{2}
$$

where $\varphi$ is the deviation angle from the optimal orientation and $c$ is a constant. Whereas $c$ is negative in usual spring-type gravimeters, it is positive in the SG. Because the vertical restoring force as given by Eq. (12) decreases for finite $r$, tilting the SG causes a horizontal displacement of the sphere from the central axis and therefore a downward motion of the sphere, i.e., an apparent increase in the gravity (GWR Instruments 1985). This means that $\beta_{\mathrm{H}}>0$. When the gravimeter is tilted from the local vertical by a small angle $\varphi$, the sphere experiences additional forces $m g \sin \varphi$ and $m g(1-\cos \varphi)$ in the horizontal and vertical directions, respectively, where $g$ is the gravity acceleration. Then, the balance of the sphere is expressed as

$$
\begin{aligned}
& -\alpha_{\mathrm{H}} r-\beta_{\mathrm{H}} r z+m g \varphi=0 \\
& -\alpha_{\mathrm{V}} z-\frac{1}{2} \beta_{\mathrm{H}} r^{2}-\frac{1}{2} \beta_{\mathrm{V}} z^{2}+\frac{1}{2} m g \varphi^{2}=0
\end{aligned}
$$

where we approximate $\sin \varphi \sim \varphi$ and $1-\cos \varphi \sim(1 / 2) \varphi^{2}$. Equation (14) indicates that $r$ is on the first order of $\varphi$. Additionally, as observed from Eq. (15), $z$ is on the second order of $\varphi$. Therefore, to the first order of $\varphi$,

$$
r=\frac{m g \varphi}{\alpha_{\mathrm{H}}}
$$

is the radial displacement of the sphere. Substituting Eq. (16) into Eq. (15), we obtain the vertical displacement as

$$
z=\frac{m g}{2 \alpha_{\mathrm{V}}}\left(1-\frac{\beta_{\mathrm{H}} m g}{\alpha_{\mathrm{H}}^{2}}\right) \varphi^{2}
$$

to the second order of $\varphi$. The change in the vertical force (upward positive) sensed by the gravimeter is given by $\alpha_{\mathrm{V}} z$, and thus, the change in the upward acceleration $\Delta a_{z}$ is

$$
\Delta a_{z}=\frac{\alpha_{\mathrm{V}} z}{m}=\frac{g}{2}\left(1-\frac{\beta_{\mathrm{H}} m g}{\alpha_{\mathrm{H}}^{2}}\right) \varphi^{2}
$$

When a feedback control on the gravity is enabled, an additional magnetic field generated by the feedback coil exerts a force on the sphere so that it cancels any external forces and the sphere is consequently held in a fixed position. Because tilt adjustment deals only with static signals, both the external force and the feedback force are regarded as temporally constant, and thus, there is no vertical motion of the sphere. This feedback force is equal in magnitude to the change in gravity to be measured. The change in the gravity acceleration is given by

$$
\Delta g=-\Delta a_{z}=\frac{g}{2}\left(\frac{\beta_{\mathrm{H}} m g}{\alpha_{\mathrm{H}}^{2}}-1\right) \varphi^{2}
$$

Comparing this with Eq. (13), we have

$$
c=\frac{g}{2}\left(\frac{\beta_{\mathrm{H}} m g}{\alpha_{\mathrm{H}}^{2}}-1\right)
$$

The actual value of the coefficient $c$ can be estimated experimentally. Figure 4 shows a result of the tilt adjustment for CT \#036. The parameter Tilt Reset determines the null position of the tilt sensor. Note that a positive change in the output voltage corresponds to a negative change in the gravity for the SG. We search for the optimal setting of Tilt Reset for each of the two tilt sensors, which we shall call $x$ and $y$. Fitting a quadratic function to the gravity changes with Tilt Reset as a free variable gives the estimate of $c$ as the optimal coefficient of the secondorder term. The results are as follows:

$$
\begin{aligned}
& c=7.105 \pm 0.068 \times 10^{-6} \mathrm{~V} \mathrm{digit}^{-2} \\
& c=8.137 \pm 0.018 \times 10^{-6} \mathrm{~V} \mathrm{digit}^{-2}
\end{aligned}
$$


where digit denotes the unit of Tilt Reset. The two values need not coincide with each other, because the effective sensitivities of Tilt Reset for $x$ and $y$ can be different. An additional file describes the calibration of sensitivity of Tilt Reset (see Additional file 1). The results of calibration are $4.189 \pm 0.005 \times 10^{-6}$ and $4.746 \pm 0.009 \times 10^{-6} \mathrm{rad} /$ digit for $x$ and $y$, respectively. Note that this calibration is different from the calibration of Tilt Power (Riccardi et al. 2009) with the automatic leveling function enabled. Using these results as well as the scale factor of this gravimeter $\left(1 \mathrm{~V}=-1134.6 \times 10^{-8} \mathrm{~ms}^{-2}\right)$, the above values of $c$ are translated into

$$
\begin{aligned}
& c=4.594 \pm 0.044 \mathrm{~ms}^{-2} \\
& c=4.099 \pm 0.009 \mathrm{~ms}^{-2}
\end{aligned}
$$

These estimates are different by approximately $12 \%$, suggesting that the supporting magnetic field may be azimuthally asymmetric. Here, neglecting this difference, we assume that there is no dependence of the coefficient $c$ on the azimuth. By taking a simple average of both $x$ and $y$, we obtain

$$
c=4.35 \mathrm{~ms}^{-2}
$$

as the estimate of $c$ for this gravimeter.

The gravity acceleration at the gravimeter pier of the VERA Ishigakijima station measured using an absolute gravimeter is $g=9.79002515 \mathrm{~ms}^{-2}$ (Miyakawa, personal communication). From Eqs. (20) and (21), we have

$$
\frac{\beta_{\mathrm{H}} m}{\alpha_{\mathrm{H}}^{2}}=0.193 \mathrm{~m}^{-1} \mathrm{~s}^{2}
$$

\section{Dynamic effects of horizontal acceleration}

Thus far, we have dealt with the static equilibrium of forces in the gravity sensor. Now, we will consider dynamic cases where external acceleration is applied to the gravimeter pier so that inertial forces are exerted on the superconducting sphere. The potential in Eq. (4) can be rewritten in the rectangular coordinate system as

$$
\begin{aligned}
U(x, y, z)= & U(0,0,0)+\frac{1}{2}\left[\alpha_{\mathrm{H}}\left(x^{2}+y^{2}\right)+\alpha_{\mathrm{V}} z^{2}\right] \\
& +\frac{1}{6}\left[3 \beta_{\mathrm{H}}\left(x^{2}+y^{2}\right) z+\beta_{\mathrm{V}} z^{3}\right]
\end{aligned}
$$

Let $X, Y, Z$ be the displacement of the pier in the inertial frame, and $x, y, z$ the displacement of the sphere with respect to the gravimeter. Then, the equations of motion of the sphere are

$$
m \ddot{x}+2 m h_{\mathrm{H}} \omega_{\mathrm{H}} \dot{x}+\alpha_{\mathrm{H}} x=-m \ddot{X}
$$
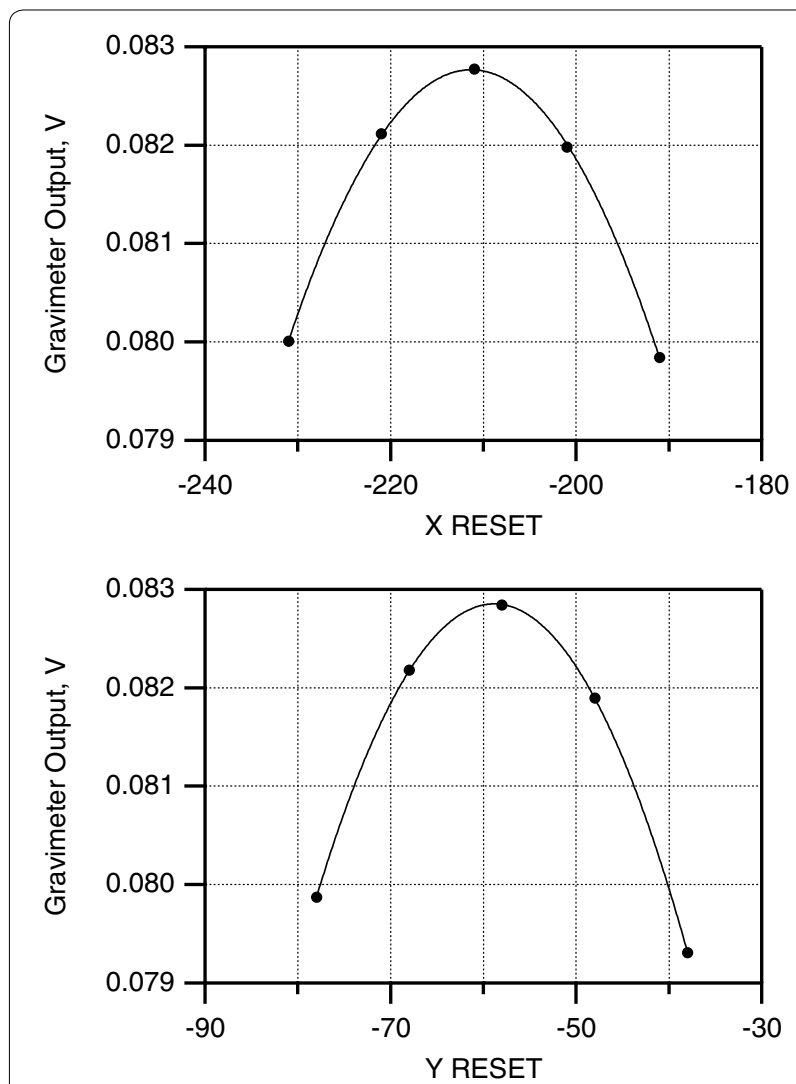

Fig. 4 Results of tilt adjustment for the superconducting gravimeter CT \#036

$$
\begin{aligned}
& m \ddot{y}+2 m h_{\mathrm{H}} \omega_{\mathrm{H}} \dot{y}+\alpha_{\mathrm{H}} y=-m \ddot{Y} \\
& m \ddot{z}+2 m h_{\mathrm{V}} \omega_{\mathrm{V}} \dot{z}+\alpha_{\mathrm{V}} z \\
& \quad+\frac{1}{2} \beta_{\mathrm{H}}\left(x^{2}+y^{2}\right)+\frac{1}{2} \beta_{\mathrm{V}} z^{2}=-m \ddot{Z}
\end{aligned}
$$

where we have retained the terms up to the first order in the horizontal components and the terms up to the second order in the vertical component. In addition, we assume that the sphere is subject to frictional forces due to eddy currents in the gravity sensor whose magnitude is proportional to the velocity of the sphere. The coefficients of the friction are $2 m h_{\mathrm{H}} \omega_{\mathrm{H}}$ and $2 m h_{\mathrm{V}} \omega_{\mathrm{V}}$ for the horizontal and vertical components, respectively. Here, $h_{\mathrm{H}}$ and $h_{\mathrm{V}}$ are dimensionless positive constants. Whereas $h_{\mathrm{V}}$ can be directly measured as described later, the value of $h_{\mathrm{H}}$ is unknown. Here, we assume that the friction has no dependence on the direction of the sphere velocity, and thus, $2 m h_{\mathrm{H}} \omega_{\mathrm{H}}=2 m h_{\mathrm{V}} \omega_{\mathrm{V}}$. Given this, Eqs. (24)-(26) can be rewritten as

$$
\ddot{x}+2 \eta \dot{x}+\omega_{\mathrm{H}}^{2} x=-\ddot{X}
$$




$$
\begin{gathered}
\ddot{y}+2 \eta \dot{y}+\omega_{\mathrm{H}}^{2} y=-\ddot{Y} \\
\ddot{z}+2 \eta \dot{z}+\omega_{\mathrm{V}}^{2} z+\frac{1}{2} \frac{\beta_{\mathrm{H}}}{m}\left(x^{2}+y^{2}\right)+\frac{1}{2} \frac{\beta_{\mathrm{V}}}{m} z^{2}=-\ddot{Z}
\end{gathered}
$$

where we define

$$
\eta=h_{\mathrm{H}} \omega_{\mathrm{H}}=h_{\mathrm{V}} \omega_{\mathrm{V}}
$$

The mechanical eigenfrequencies of the mass-spring system are given by

$$
\begin{aligned}
& f_{\mathrm{H}}=\frac{\omega_{\mathrm{H}}}{2 \pi} \\
& f_{\mathrm{V}}=\frac{\omega_{\mathrm{V}}}{2 \pi}
\end{aligned}
$$

for the horizontal and vertical directions, respectively. $\omega_{\mathrm{H}} \gg \omega_{\mathrm{V}}$ means $f_{\mathrm{H}} \gg f_{\mathrm{V}}$. The eigenfrequencies are dependent on the user-adjustable coil currents and therefore are not intrinsic to the particular instrument. Typically, $f_{\mathrm{V}}$ is on the order of $0.1 \mathrm{~Hz}$, and it can be measured by applying external vertical forces on the sphere (Imanishi et al. 1996; Van Camp et al. 2000). Meanwhile, there is no easy way to directly measure $f_{\mathrm{H}}$ experimentally.

Before the SG was moved to Ishigakijima, we measured its open-loop and closed-loop transfer functions at Tsukuba University (Ikeda et al. 2013). Sinusoidal functions with $1 \mathrm{~V}$ amplitude at nine discrete frequencies from 0.002 to $1 \mathrm{~Hz}$ were applied to the feedback coil of the SG to measure the responses. The vertical eigenfrequency and dissipation parameter obtained from this experiment are $f_{\mathrm{V}}=0.120 \mathrm{~Hz}$ and $h_{\mathrm{V}}=6.41$. Although we have not performed a similar experiment at Ishigakijima, we can estimate the present value of the eigenfrequency as follows. When adjusting the supporting magnetic field, we measure the displacement of the levitating sphere in response to a constant vertical force generated by a $10 \mathrm{~mA}$ current. This is called the "magnetic gradient," a parameter that corresponds to the inverse of the stiffness of the spring. The final values of the magnetic gradient were $1.613 \mathrm{~V} / 10 \mathrm{~mA}$ at Tsukuba and $1.834 \mathrm{~V} / 10 \mathrm{~mA}$ at Ishigakijima. Since the magnetic gradient is inversely proportional to $\alpha_{\mathrm{V}}$, it follows from the final gradients that the value of $\alpha_{\mathrm{V}}$ at Ishigakijima is equal to 0.879 times that at Tsukuba. Considering that the eigenfrequency is proportional to the square root of $\alpha_{\mathrm{V}}$, $f_{\mathrm{V}}$ at Ishigakijima should be equal to $0.112 \mathrm{~Hz}$. Also, considering that the dissipation is provided by the currents induced in the non-superconducting parts of the gravity sensor, we assume that the parameter $\eta$ is independent of the magnetic gradient. Therefore, the value at Tsukuba

$$
\eta=2 \pi h_{\mathrm{V}} f_{\mathrm{V}}=4.833 \mathrm{~s}^{-1}
$$

also applies to Ishigakijima.

The response of this dynamic system to an external acceleration in the $x$ direction is described in the frequency domain as

$$
\frac{\tilde{x}}{\tilde{X}}=\frac{\omega^{2}}{-\omega^{2}+2 i \eta \omega+\omega_{\mathrm{H}}^{2}}
$$

where $\omega$ is the angular frequency and $\tilde{x}$ and $\tilde{X}$ are the Fourier transforms of $x$ and $X$, respectively. A similar equation holds also for the $y$ component. Note that there is no feedback control in the horizontal directions. The sphere is displaced horizontally according to the response in Eq. (34), resulting in a change in the mean vertical position of the sphere through the term containing $\beta_{\mathrm{H}}$ in Eq. (26). Denoting a temporal average as \langle\rangle we have

$$
\langle z\rangle=-\frac{1}{2} \frac{\beta_{\mathrm{H}}}{\alpha_{\mathrm{V}}}\left[\left\langle x^{2}\right\rangle+\left\langle y^{2}\right\rangle\right]
$$

where we neglect the higher-order term of $z$ (the term containing $\beta_{\mathrm{V}}$ ). Because $\alpha_{\mathrm{V}}>0$ and $\beta_{\mathrm{H}}>0$, the righthand side of Eq. (35) is negative, and thus, the mean position of the sphere moves downward. This is observed as an increase in the DC component of the gravity acceleration given by

$$
\Delta g=-\frac{\alpha_{\mathrm{V}}}{m} z=\frac{1}{2} \frac{\beta_{\mathrm{H}}}{m}\left[\left\langle x^{2}\right\rangle+\left\langle y^{2}\right\rangle\right]
$$

This implies that the mean-squared horizontal displacements of the sphere are proportional to the apparent change in the gravity acceleration. In particular, if the input acceleration has a single angular frequency $\sigma$, then

$$
\frac{x}{X}=\frac{\sigma^{2}}{-\sigma^{2}+2 i \eta \sigma+\omega_{\mathrm{H}}^{2}}
$$

holds in the time domain, and therefore, we have

$$
\Delta g=\frac{1}{2} \frac{\beta_{\mathrm{H}}}{m}\left|\frac{\sigma^{2}}{-\sigma^{2}+2 i \eta \sigma+\omega_{\mathrm{H}}^{2}}\right|^{2}\left[\left\langle X^{2}\right\rangle+\left\langle Y^{2}\right\rangle\right]
$$

We use the horizontal acceleration data caused by the motion of the VLBI antenna to verify whether this theoretical prediction is correct. Figure 5 shows a typical example of the ground vibrations induced by the antenna and the resultant disturbances in the gravity recordings. The seismometer used to record the ground vibrations is an $\mathrm{L}-4 \mathrm{C} 1.0-\mathrm{Hz}$ three components velocity transducer, manufactured by Mark Products, that was placed on the gravimeter pier (Ohtaki and Nawa 2013). The raw data shown in Fig. 5 were converted into ground displacements by deconvolving the transfer functions of 
the sensors. We can see from Fig. 5 that the north-south and east-west components have similar magnitudes of power, although they are variable with time, whereas the power of the up-down component is only $1 \%$ of the magnitudes of the horizontal components. Figure 6 shows the power spectra of the three components. The spectral peaks at $4.92 \mathrm{~Hz}$ and at its harmonics found in all the components are due to the movements of the antenna. It is unknown which part of the antenna is responsible for the observed frequencies. The power ratio for the peaks at 4.92 and $9.84 \mathrm{~Hz}$ is approximately 117 and 179 for the north-south and east-west components, respectively. Therefore, we regard the vibration as monochromatic in the following analysis.

The positive steps in the gravity signals during the period of antenna movement as seen in Fig. 5 are collected and compared with the ground displacements in Fig. 7. The ground displacements are displayed in terms of time-domain variances. Note that the above-mentioned $x$ and $y$ of the tilt sensors of the SG are not oriented in the east-west and north-south directions. For each north-south, east-west and up-down component (Fig. 7a, b, d), respectively), ground displacements and gravity changes appear to be correlated with linear correlation coefficients 0.78-0.90. Meanwhile, the linear correlation is clearer with the correlation coefficient as high as 0.97 in the case of the sum of the north-south and east-west components, i.e., the horizontal component (Fig. 7c). This fact provides evidence that the assumptions for our theoretical model are appropriate. In the following, we consider only the horizontal components of ground vibrations.

From Eq. (38) and considering the proportionality shown in Fig. 7c, we obtain

$$
\frac{1}{2} \frac{\beta_{\mathrm{H}}}{m}\left|\frac{\sigma^{2}}{-\sigma^{2}+2 i \eta \sigma+\omega_{\mathrm{H}}^{2}}\right|^{2}=1.36 \times 10^{4} \mathrm{~m}^{-1} \mathrm{~s}^{-2}
$$

where $\sigma=2 \pi \times 4.92=30.9 \mathrm{~s}^{-1}$. By defining two new variables $\alpha_{\mathrm{H}}^{\prime}$ and $\beta_{\mathrm{H}}^{\prime}$ as

$$
\begin{aligned}
& \alpha_{\mathrm{H}}^{\prime}=\frac{\alpha_{\mathrm{H}}}{m} \\
& \beta_{\mathrm{H}}^{\prime}=\frac{\beta_{\mathrm{H}}}{m}
\end{aligned}
$$

Eqs. (22) and (39) can be rewritten as

$$
\frac{\beta_{\mathrm{H}}^{\prime}}{\left(\alpha_{\mathrm{H}}^{\prime}\right)^{2}}=0.193 \mathrm{~m}^{-1} \mathrm{~s}^{2}
$$

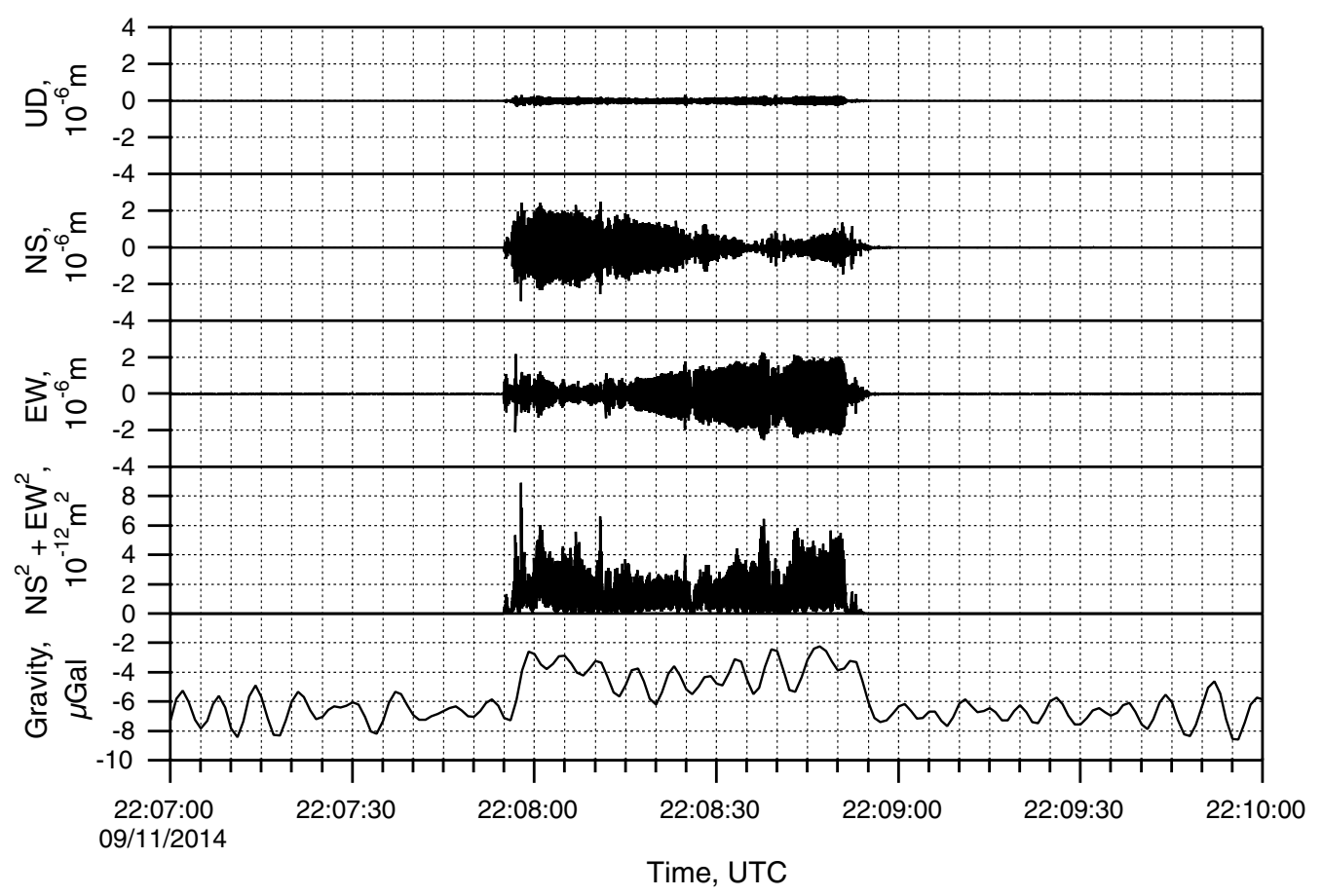

Fig. 5 Ground displacements caused by movements of the VLBI antenna recorded by a seismometer and the simultaneous offset in the gravity. UD, NS and EW denote the up-down, north-south and east-west components, respectively. $\left(\mathrm{NS}^{2}+\mathrm{EW}^{2}\right)$ is also shown for comparison with the gravity 


$$
\frac{1}{2} \beta_{\mathrm{H}}^{\prime}\left|\frac{\sigma^{2}}{-\sigma^{2}+2 i \eta \sigma+\alpha_{\mathrm{H}}^{\prime}}\right|^{2}=1.36 \times 10^{4} \mathrm{~ms}^{-1}
$$

Together with Eq. (33), Eqs. (42) and (43) can be solved for $\alpha_{\mathrm{H}}^{\prime}$ and $\beta_{\mathrm{H}}^{\prime}$ as

$$
\begin{aligned}
& \alpha_{\mathrm{H}}^{\prime}=2.88 \times 10^{2} \mathrm{~s}^{-2} \\
& \beta_{\mathrm{H}}^{\prime}=1.60 \times 10^{4} \mathrm{~m}^{-1} \mathrm{~s}^{-2}
\end{aligned}
$$

Therefore,

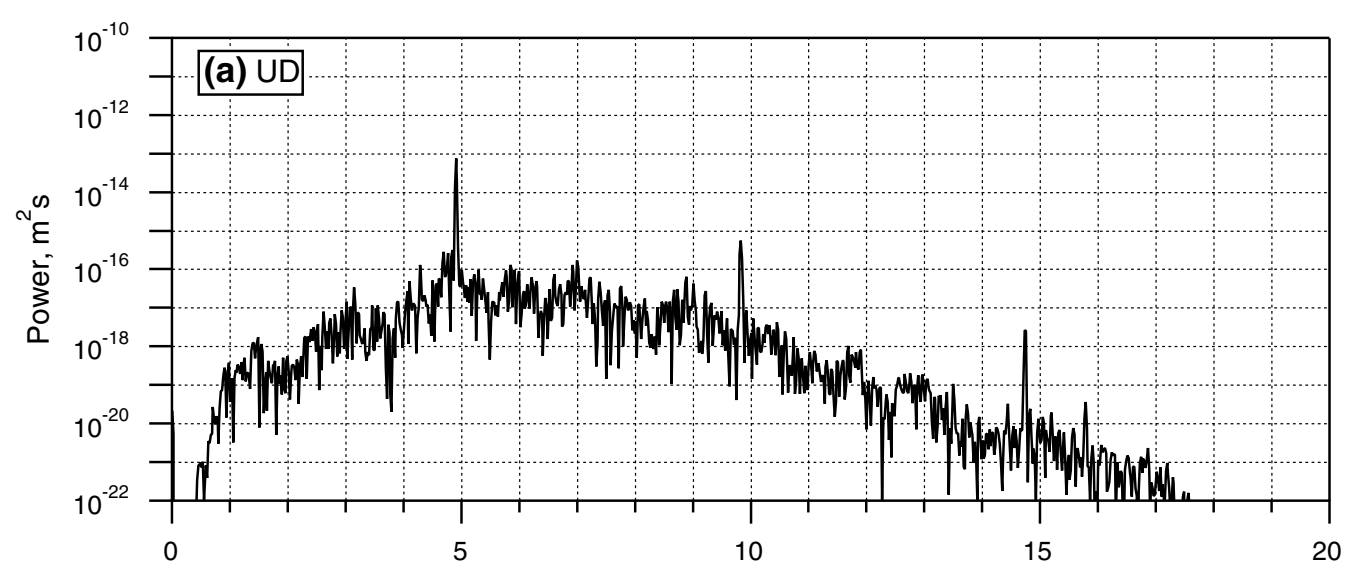

Frequency, $\mathrm{Hz}$

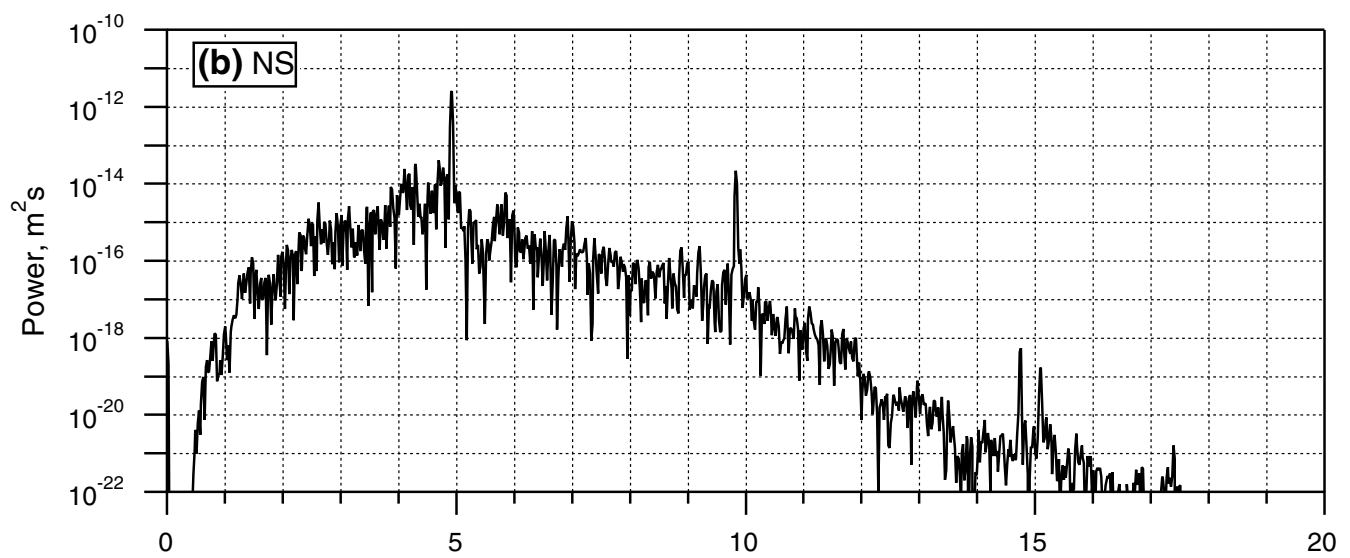

Frequency, $\mathrm{Hz}$

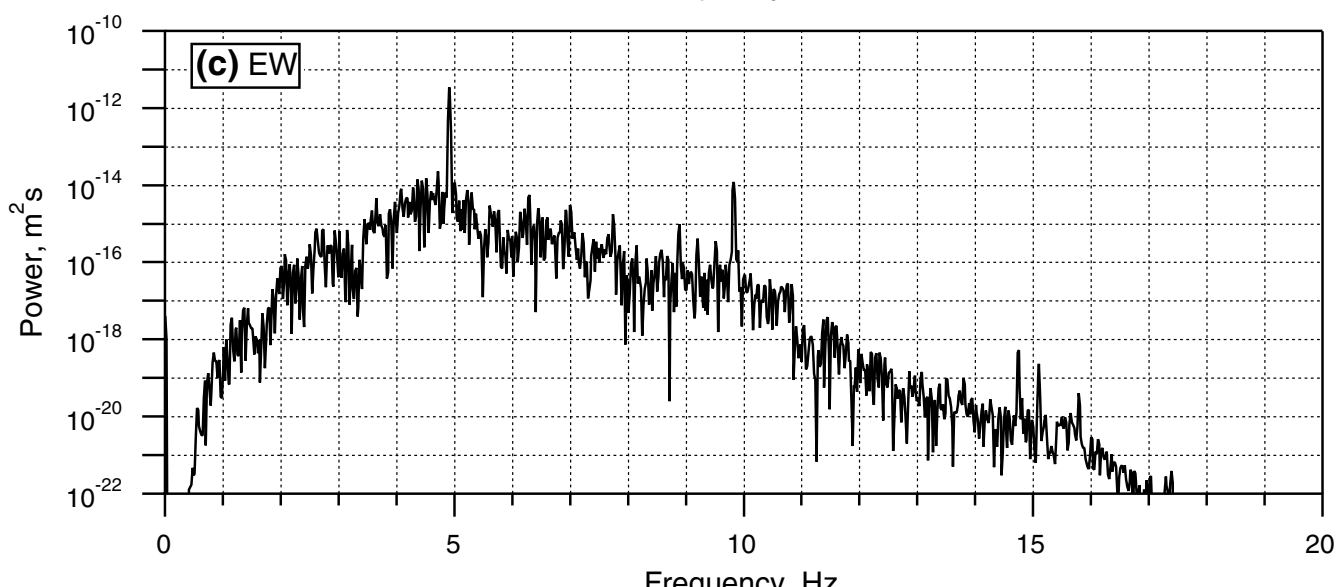

Frequency, $\mathrm{Hz}$

Fig. 6 Power spectra of the ground displacement recorded by a seismometer. $\mathbf{a}$ Up-down component, $\mathbf{b}$ north-south component and $\mathbf{c}$ eastwest component. This was acquired while the VLBI antenna was moving 


$$
\begin{aligned}
& \omega_{\mathrm{H}}=\sqrt{\alpha_{\mathrm{H}}^{\prime}}=17.0 \mathrm{~s}^{-1} \\
& f_{\mathrm{H}}=\frac{\omega_{\mathrm{H}}}{2 \pi}=2.70 \mathrm{~s}^{-1} \\
& h_{\mathrm{H}}=0.285
\end{aligned}
$$

In the above result, there is no assumption regarding the value of $m$.

The most uncertain parameter in the above analysis is $\eta$, for which we have assumed the same value $\left(\eta=4.833 \mathrm{~s}^{-1}\right)$ as measured at Tsukuba. Figure 8 shows the dependence of the estimates of $\alpha_{\mathrm{H}}^{\prime}$ and $\beta_{\mathrm{H}}^{\prime}$ on the variable $\eta$. Both $\alpha_{\mathrm{H}}^{\prime}$ and $\beta_{\mathrm{H}}^{\prime}$ increase monotonically as $\eta$ increases. In the extreme case where there is no dissipation $(\eta=0)$, we obtain $\alpha_{\mathrm{H}}^{\prime}=2.70 \times 10^{2} \mathrm{~s}^{-2}$ and $f_{\mathrm{H}}=2.61 \mathrm{~s}^{-1}$. If the dissipation is approximately twice as large $(\eta=10)$ as our previous assumption, we have $\alpha_{H}^{\prime}=3.43 \times 10^{2} \mathrm{~s}^{-2}$ and $f_{\mathrm{H}}=2.95 \mathrm{~s}^{-1}$. Therefore, we can conclude that $f_{\mathrm{H}}$ of this gravimeter is approximately $3 \mathrm{~Hz}$ for a practically plausible range of the dissipation parameter.

Thus, we have successfully modeled the effects of horizontal acceleration due to the motion of the VLBI antenna on the SG quantitatively. As a by-product of the model analysis, the horizontal eigenfrequency $f_{\mathrm{H}}$ of the mass-spring system was indirectly estimated. It is noted that the precondition for the analysis $\left(f_{\mathrm{H}} \gg f_{\mathrm{V}}\right)$ is satisfied because $f_{\mathrm{V}}$ is on the order of $0.1 \mathrm{~Hz}$.
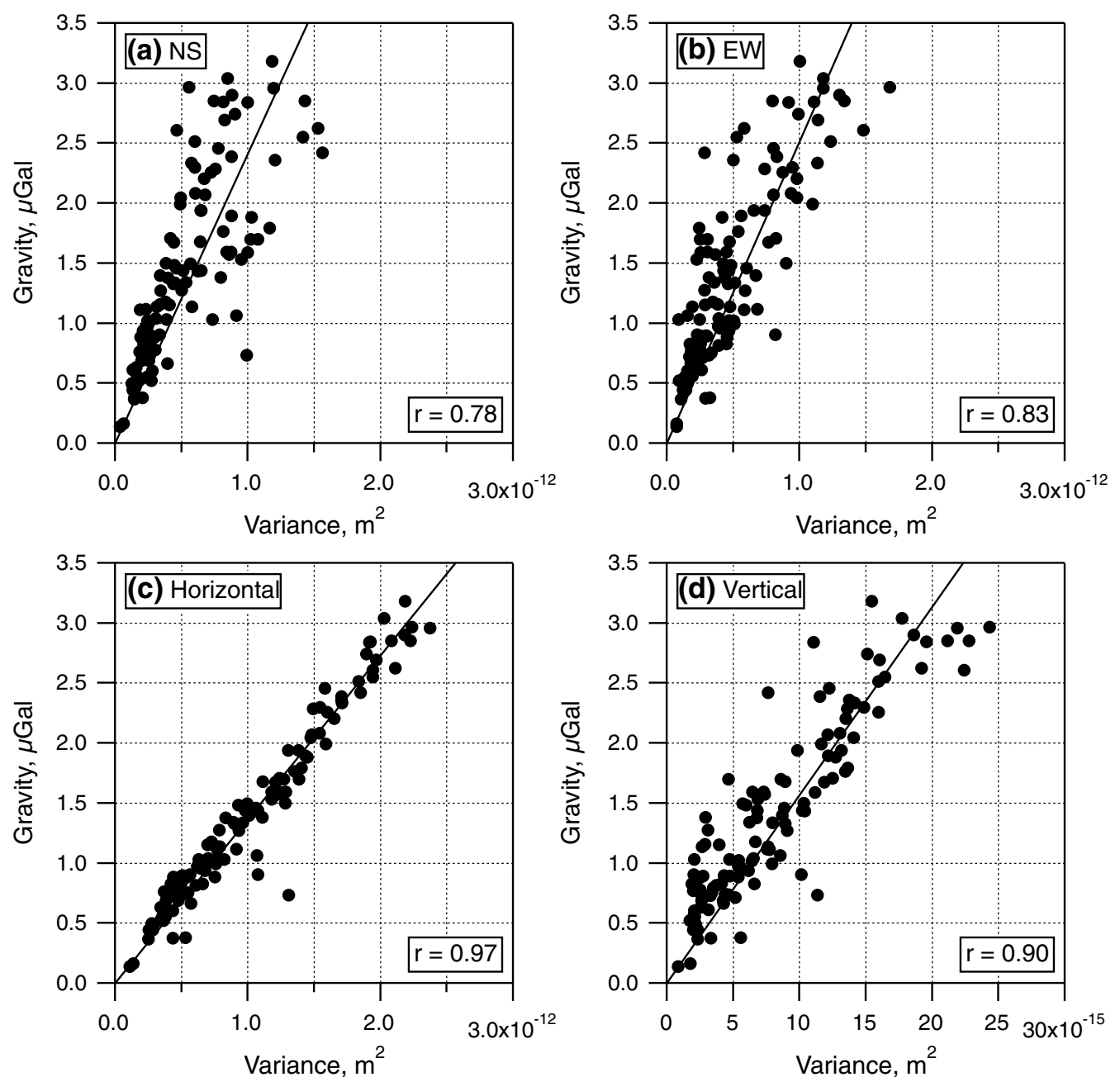

Fig. 7 Relationship between the ground displacements and gravity changes. The four panels represent the a north-south component, $\mathbf{b}$ eastwest component, $\mathbf{c}$ horizontal component and $\mathbf{d}$ vertical component. The ground displacements are shown in terms of variances in time domain. Straight lines are the results of linear regression for the data, and the linear correlation coefficients are also shown. Note that the horizontal scale in d is different from those in the other panels 


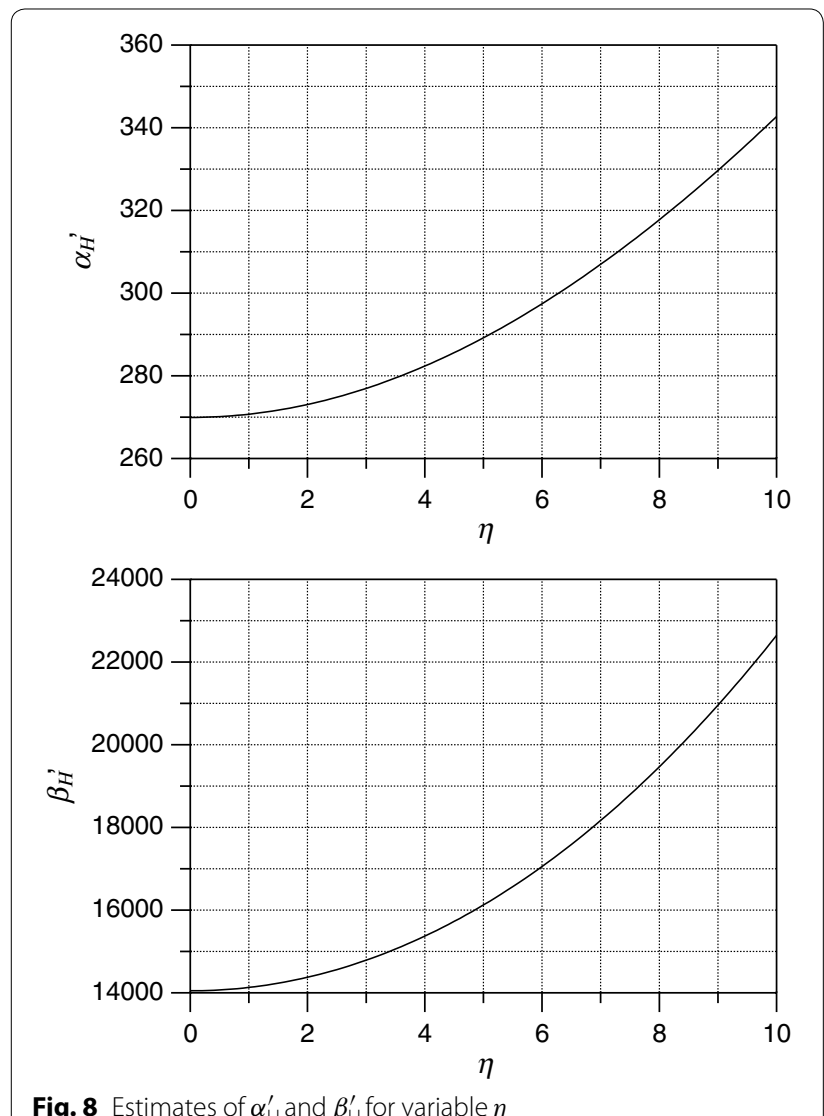

Fig. 8 Estimates of $\alpha_{H}^{\prime}$ and $\beta_{H}^{\prime}$ for variable $\eta$

\section{Discussion}

The influence of a collocated VLBI antenna on the SG was already investigated by H.-G. Scherneck for the Onsala Space Observatory, Sweden (http://holt.oso.chalmers.se/hgs/SCG/AntennaPointing.html). His work was focused on the effect of direct Newtonian attraction by the mass of the antenna, rather than the effect of induced ground vibrations. The conclusion was that the attraction effect was negligible at the Onsala station due to the long distance $(80 \mathrm{~m})$ between the VLBI antenna and the gravimeter (Scherneck, personal communication). Following his method of analysis, we assessed the attraction effect for our station. Approximating the antenna with a parabolic dish and a counterweight (each weighing 200 tons), the maximal possible change in the direct attraction is approximately $0.08 \mu \mathrm{Gal}$. This is negligible in our present context. Mizusawa (Japan) is another station of the VERA project where both a VLBI antenna and an SG (TT \#016) are located. At Mizusawa, the effects of the VLBI antenna (either direct attraction or ground vibrations) on the gravimeter are almost invisible, again due to the long distance $(70 \mathrm{~m})$ between them.
When deriving Eqs. (35) from (26) in "Effects of movement of the VLBI antenna on the gravimeter" section, we ignored the term containing $\beta_{\mathrm{V}}$ in the left-hand sides of Eqs. (26) or (29). This term originates in the third-order term of $z$ in the expansion of the potential in Eq. (1). When analyzing the effect of the VLBI antenna on the gravimeter, effects of this term were negligible because the vertical displacement of the sphere may be regarded as small. On the other hand, the vertical displacement of the sphere can be large during stormy weather, because the level of ground noise is extremely enhanced than usual. In such a condition, the third-order term of $z$ might have some effect, possibly giving rise to additional offsets in observed gravity signals. Indeed, we observe such gravity changes that cannot be explained solely by atmospheric effects when typhoons (hurricanes) approach the Ishigakijima Island. Investigation of this effect requires experimental determination of the coefficient $\beta_{\mathrm{V}}$.

The horizontal mass-spring system as a harmonic oscillator in Eqs. (24) or (25) is called underdamped if $h_{\mathrm{H}}<1$ and overdamped if $h_{\mathrm{H}}>1$. Our present estimate of the dissipation parameter $h_{\mathrm{H}}$ given in Eq. (48) means that the system is underdamped. Figure 9 shows the magnitude of the right-hand side of Eq. (34) using the estimated parameters. We can see that the magnitude response is slightly enhanced near the horizontal eigenfrequency. Although this will not affect the performance of the gravimeter at quiet stations, it may have some effect on the signal-tonoise ratio of gravity recordings at relatively noisy sites such as Ishigakijima. In particular, the magnitude response at $5 \mathrm{~Hz}$ is 1.29 , implying that the ground displacements induced by the movements of the VLBI antenna are amplified by approximately 30 per cent in the horizontal displacements of the sphere. To mitigate this effect, either the horizontal eigenfrequency $f_{\mathrm{H}}$ or the dissipation parameter $h_{\mathrm{H}}$, or both, must be increased significantly. This fact should be taken into account in future development of gravity sensors based on magnetic suspension.

Figure 9 also shows the mechanical response of CT \#036 for the vertical direction. The SG as a mechanical pendulum system is overdamped in the vertical direction (Imanishi et al. 1996). The two curves intersect at approximately $2 \mathrm{~Hz}$. In other words, the response of the superconducting sphere to applied acceleration of unit magnitude in the horizontal direction is of similar magnitude to that in the vertical direction at $2 \mathrm{~Hz}$. In this sense, this frequency may be regarded as an upper limit of the frequency range in which the SG is able to work properly as a gravity meter. Practically, the upper limit at a given station will depend on the spectra of background noise for the horizontal and vertical components as well as the magnitude of the parameters $\alpha_{\mathrm{H}}, \beta_{\mathrm{H}}$ and $\eta$. 


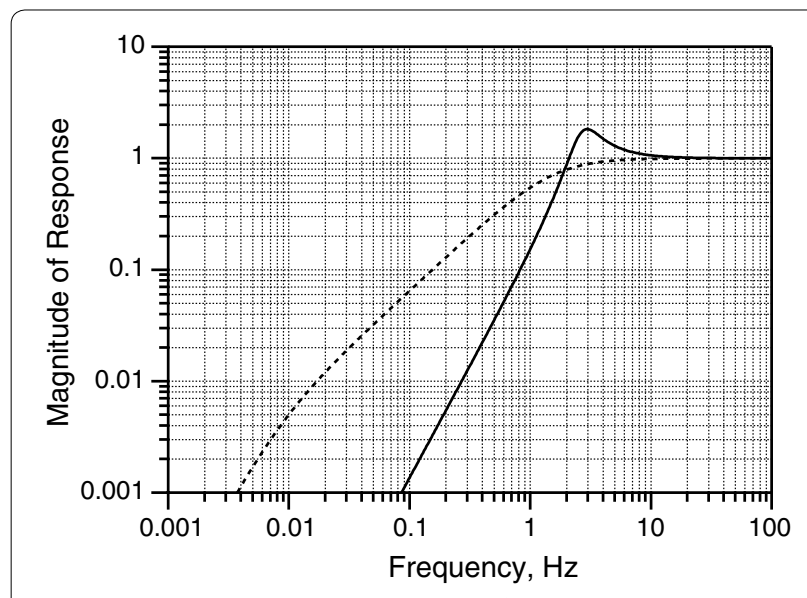

Fig. 9 Theoretical magnitude responses of the mass-spring systems for the superconducting gravimeter CT \#036 in the horizontal (solid line) and vertical (broken line) directions

\section{Conclusions}

In this paper, we investigated the possible effects of horizontal acceleration on the SG operated at Ishigakijima from the viewpoint of a gravity sensor based on magnetic suspension. We developed a theoretical model considering the coupling between the horizontal and vertical components derived from the potential of the sphere to explain the step changes caused by the input of a $5-\mathrm{Hz}$ horizontal acceleration excited by the motion of the VLBI antenna. Numerical analysis using the data from a collocated seismometer proved that this model could effectively explain the behavior of the gravimeter. It was also shown that the superconducting sphere levitated in the gravity sensor has a horizontal eigenfrequency of approximately $3 \mathrm{~Hz}$. The latter fact implies that the gravity sensor could be susceptible to horizontal acceleration at this particular frequency. It may be advisable to keep $3-\mathrm{Hz}$ horizontal acceleration low so that the coupling between the horizontal and vertical components does not degrade the quality of gravity recordings.

Here we were focused on the effects of horizontal acceleration on the gravity sensor of the SG and resultant apparent gravity changes through the coupling between the horizontal and vertical components. Effects of threedimensional acceleration induced by enhanced ground noise, typically seen when typhoons approach Ishigakijima, will be studied in the future taking into account also the third-order term of $z$ in the expansion of the potential.

\section{Additional file}

Additional file 1. Calibration of tilt sensors and Tilt Reset for the superconducting gravimeter $\mathrm{CT} \# 036$.

\section{Abbreviations}

SG: Superconducting gravimeter; VERA: VLBI exploration of radio astrometry.

\section{Authors' contributions}

YI developed the theory, conducted the data analysis and interpreted the results. KN made the observations with seismometers and prepared the data. $\mathrm{YT}$ and $\mathrm{HI}$ installed and maintained the superconducting gravimeter. All authors read and approved the final manuscript.

\section{Author details \\ ${ }^{1}$ Earthquake Research Institute, The University of Tokyo, 1-1-1, Yayoi, Bunkyo, Tokyo 113-0032, Japan. ${ }^{2}$ Geological Survey of Japan, National Institute of Advanced Industrial Science and Technology, AIST Tsukuba Central 7, 1-1-1 Higashi, Tsukuba, Ibaraki 305-8567, Japan. ${ }^{3}$ Mizusawa VLBI Observa- tory, National Astronomical Observatory of Japan, 2-12 Hoshigaoka-cho, Mizusawa-ku, Oshu, Iwate 023-0861, Japan. ${ }^{4}$ Research Facility Center for Sci- ence and Technology, University of Tsukuba, 1-1-1 Tennodai, Tsukuba, Ibaraki 305-8577, Japan.}

\section{Acknowledgements}

We are grateful to the VERA project of the National Astronomical Observatory of Japan for supporting our superconducting gravimeter observations at the VERA Ishigakijima station. T. Okuda of Nagoya University kindly allowed us to use the seismometer at Ishigakijima. We thank the editor and two anonymous reviewers for their careful reading and constructive comments. This work was financially supported by JSPS KAKENHI Grant Numbers JP23340125, JP26289350 and JP26610139, and by the Cooperative Research Program of Earthquake Research Institute, The University of Tokyo.

\section{Competing interests}

The authors declare that they have no competing interests.

\section{Availability of data and materials}

The data from gravimeter, seismometer, and so on, used in this paper will not be shared, because successive works of our own using them are under way.

\section{Consent for publication \\ Not applicable.}

\section{Ethics approval and consent to participate}

Not applicable.

\section{Publisher's Note}

Springer Nature remains neutral with regard to jurisdictional claims in published maps and institutional affiliations.

Received: 20 September 2017 Accepted: 2 January 2018

Published online: 12 January 2018

\section{References}

Goodkind JM (1999) The superconducting gravimeter. Rev Sci Instrum 70:4131-4152

GWR Instruments (1985) Superconducting gravity meter model TT70 operating manual. GWR Instruments, San Diego

Heki K, Kataoka T (2008) On the biannualy repeating slow slip events at the Ryukyu Trench, Southwest Japan. J Geophys Res 113:B11402. https://doi. org/10.1029/2008JB005739

Hinderer J, Crossley D, Warburton RJ (2015) Superconducting gravimetry. In: Schubert G (ed) Treatise on geophysics, 2nd edn, vol 3. Elsevier, Oxford, pp 59-115

Honma M et al (2000) J-net galactic-plane survey of VLBI radio sources for VLBI exploration of radio astrometry (VERA). Publ Astron Soc Jpn 52:631-643

Ikeda H, Nawa K, Imanishi Y (2013) Refurbishment and performance of the superconducting gravimeter CT-36. J Geod Soc Jpn 59:25-36

ImanishiY (2005) On the possible cause of long period instrumental noise (parasitic mode) of a superconducting gravimeter. J Geod 78:683-690 
Imanishi Y (2009) High-frequency parasitic modes of superconducting gravimeters. J Geod 83:455-467

Imanishi Y, Sato T, Asari K (1996) Measurement of mechanical responses of superconducting gravimeters. J Geod Soc Jpn 42:115-117

Nawa K, Suda N, Yamada I, Miyajima R, Okubo S (2009) Coseismic change and precipitation effect in temporal gravity variation at Inuyama, Japan: a case of the 2004 off the Kii peninsula earthquakes observed with a superconducting gravimeter. J Geodyn 48:1-5

Ohtaki T, Nawa K (2013) Rough estimate of P-wave velocity beneath the VERA Ishigakijima station for correcting hydrological disturbance in gravity observation data. J Geod Soc Jpn 59:147-156
Prothero WA, Goodkind JM (1968) A superconducting gravimeter. Rev Sci Instrum 39:1257-1262

Riccardi U, Hinderer J, Boy J-P, Rogister Y (2009) Tilt effects on GWR superconducting gravimeters. J Geodyn 48:316-324

Rosat S, Hinderer J, Crossley D, Boy J-P (2004) Performance of superconducting gravimeters from long-period seismology to tides. J Geodyn 38:461-476

Van Camp M, Wenzel H-G, Schott P, Vauterin P, Francis O (2000) Accurate transfer function determination for superconducting gravimeters. Geophys Res Lett 27:37-40

\section{Submit your manuscript to a SpringerOpen ${ }^{\circ}$ journal and benefit from:}

- Convenient online submission

- Rigorous peer review

- Open access: articles freely available online

- High visibility within the field

- Retaining the copyright to your article

Submit your next manuscript at $\boldsymbol{\nabla}$ springeropen.com 